4 Malhotra SL. Dietary factors in a study of cance colon from Cancer Registry with special reference to the role of saliva, milk and fermented milk products and vegetable fibre. Med Hypotheses 1977; 3: 122-6.

\section{Gastric cancer mucins as clinical markers}

EDITOR, - We read with interest the article by Hakkinen et al ${ }^{1}$ reporting the development of a new monoclonal antibody against two epitopes of mucin which they had precipitated from the gastric juice of a patient with gastric carcinoma The epitopes were expressed in fetal tissue and most cases of adult gastric carcinoma tested.

We have detected a glycoprotein fragment of molecular size 55-65 kilodaltons cofractionating with mucin in the gastric mucus (not gastric juice) of 30 patients with gastric carcinoma. ${ }^{2-}$ We also found it in 30 patients with gastric ulcers in common with Hakkinen et al. ${ }^{\prime}$ We did not detect it in the mucus from the stomachs of 16 normal subjects (eight at necropsy, eight transplant donor). In our study crude mucus scrapings were used and mucins were extracted from the gel in the presence of proteolytic inhibitors and isolated by density gradient ultracentrifugation in caesium chloride, twice for 48 hours each at $105000 \mathrm{~g}$. Mucins were then characterised by Sepharose $2 B$ and $4 B$ ge filtration and polyacrylamide gel electrophoresis in sodium dodecyl sulphate (SDSPAGE).

Mucins eluted as a carbohydrate positive peak mainly in the included volume of a
Sepharose 2B gel filtration column suggesting that they were degraded; a protein (Lowry) positive peak eluted in the near total volume of this volume under a carbohydrate (PAS) positive shoulder. Sepharose 4B gel chromatography, on the other hand, showed that this degradation of the mucins was most extensive in the cancer group, followed, in order, by ulcer and control groups. This finding was confirmed on a 4-20\% gradient gel stained with PAS

SDS-PAGE analysis of the protein positive peak in the near total volume of the Sepharose 2B column showed a glycosylated fragment of Mr 55-65 kilodaltons which was not dissimilar in size to the epitopes found by Hakkinen et al with their immunoblotting experiments; this appeared in mucins from the cancer and ulcer groups. We also note that Hakkinen et al showed a protein positive band at molecular weight approximately 67 kilodaltons, which they have not elaborated upon (Fig 2). We have also detected this band and found it to be albumin by western blot analysis using a human albumin antibody. The use of differen gel stains showed the albumin to be clearly distinguishable from the carbohydrate positive fragment.

This albumin carbohydrate complex obviously resisted dissociation in $3.5 \mathrm{M} \mathrm{CsCl}$, but could be separated partially from the mucin in sodium dodecyl sulphate at $100^{\circ} \mathrm{C}$ for one to two minutes, or by gel filtration. Treatment of mucin with $0.2 \mathrm{M}$ 2-mercaptoethanol did not produce this albumin carbohydrate complex from the control groups nor did it increase its amount in the diseased states. It has also been found in the gastric juice of a patient with cancer but was absent in the juice of patients of other groups.

We are continuing our work with gastric juice rather than mucus scrapings which are more difficult to obtain; we hope to harvest this albumin carbohydrate complex in sufficient quantities and, like Hakkinen et al, 'to raise an antibody against it and investigate its potential as a marker for premalignancy in a high risk group.

$$
\begin{array}{r}
\text { A MALL } \\
\text { H McLEOD } \\
\text { D DENT } \\
\text { R HICKMAN } \\
\text { Department of Surgery and } \\
\text { MRC Liver Research Centre, } \\
\text { Oniversity of Cape Town Medical School, } \\
\text { Observatory 7925, } \\
\text { Republic of South Africa }
\end{array}
$$

This work is supported by the National Cancer Association and Medical Research Council of South Africa.

1 Hakkinen I, Nevalainen T, Paasivuo R, Seppala K, Sipponen P. Gastric cancer associated structure in mucus glycoproteins shown as a clinically useful marker. Gut 1991; 32: 1465-9.

2 Mall A, McLeod H, Dent D; Hickman R. Biochemical characterisation of mucus in gastric carcinoma. In: Sharon N, Lis H, Duksin D Kahane I, eds. Proceedings of the Xth International Symposium of Glycoconjugates. Jerusalem, 1989.

3 Mall A, McLeod H, Jaskiewicz K, Dent D, Hickman R. Putative glycosylated 'linker' protein in gastric carcinoma. S Afr J Sci 1990; 86: tein in 6 .

4 Mall A, McLeod H, Hickman R, Dent D. Further investigations of mucins in gastric carcinoma. $S$ investigations of mucins in 